\title{
Non-commutative resolutions and Grothendieck groups
}

\author{
Hailong Dao, Osamu Iyama,* Ryo Takahashi ${ }^{\ddagger}$ and Charles Vial ${ }^{\S}$
}

\begin{abstract}
Let $R$ be a noetherian normal domain. We investigate when $R$ admits a faithful module whose endomorphism ring has finite global dimension. This can be viewed as a noncommutative desingularization of $\operatorname{Spec}(R)$. We show that the existence of such modules forces stringent conditions on the Grothendieck group of finitely generated modules over $R$. In some cases those conditions are enough to imply that $\operatorname{Spec}(R)$ has only rational singularities.
\end{abstract}

Mathematics Subject Classification (2010). 13D15, 14B05, 14E15, 16G30, $18 \mathrm{G} 20$.

Keywords. Non-commutative resolutions, Grothendieck groups, rational singularities.

\section{Introduction}

The use of non-commutative algebras with finite global dimension was initiated by M. Auslander in representation theory of Cohen-Macaulay rings, or more generally, orders $[1,2,37]$. He introduced two important classes of non-commutative algebras with finite global dimension called Auslander algebras and non-singular orders. Auslander algebras are defined as the endomorphism algebras of additive generators in the category of Cohen-Macaulay modules over representation-finite orders. Then representation theory of representation-finite orders is encoded in the structure of their Auslander algebras, and this picture was the starting point of Auslander-Reiten theory. On the other hand, the representation theory of non-singular orders is most basic since all Cohen-Macaulay modules are projective.

Recently, the study of such algebras have found striking applications in algebraic geometry. Perhaps the most well-known example is Van den Bergh's definition of non-commutative crepant resolution, usually abbreviated by NCCR ([33]). We recall Van den Bergh's definition, in a slightly more general setting: A reflexive module

\footnotetext{
*The first author was partially supported by NSF grants DMS 0834050 and DMS 1104017.

** The second author was partially supported by JSPS Grant-in-Aid for Scientific Research 24340004 , 23540045, 20244001 and 22224001.

*The third author was partially supported by JSPS Grant-in-Aid for Young Scientists (B) 22740008 and by JSPS Postdoctoral Fellowships for Research Abroad.

${ }^{\S}$ The fourth author was supported by EPSRC Postdoctoral Fellowship EP/H028870/1.
} 
$M$ over a commutative noetherian normal domain $R$ is said to give an NCCR of $\operatorname{Spec}(R)$ if $\Lambda=\operatorname{End}_{R}(M)$ is a non-singular $R$-order (i.e. $\Lambda_{\mathfrak{p}}$ is a maximal CohenMacaulay $R_{\mathfrak{p}}$-module and gl. $\operatorname{dim} \Lambda_{\mathfrak{p}}=\operatorname{dim} R_{\mathfrak{p}}$ for any $\mathfrak{p} \in \operatorname{Spec}(R)$ ).

These non-commutative objects provide a particularly pleasant explanation of the Bondal-Orlov conjecture on the derived equivalence of threefolds related by a flop. Van den Bergh's work has quickly generated a sizable body of research, see for example the recent survey $[17,24]$.

In this note we study a weaker notion which is called a non-commutative resolution $(N C R)$.

Definition 1.1. A finitely generated module $M$ over a commutative noetherian ring $R$ is said to give a NCR of $\operatorname{Spec}(R)$ (or just $R$ by abuse of notation) if $M$ is faithful and $\operatorname{End}_{R}(M)$ has finite global dimension.

The assumption that $M$ is faithful in Definition 1.1 is reasonable since, for example, any simple $R$-module $k$ gives $k=\operatorname{End}_{R}(k)$ which always has finite global dimension. Basic examples of NCRs appeared in representation theory: Auslander algebras [1], higher Auslander algebras [19, 21] and NCCRs [33]. It is known that NCRs exist when $R$ is artinian, or reduced and one-dimensional (see [24]).

We shall try to give information on the following

Question 1.2. When does R have a NCR?

We give necessary conditions which focus on the Grothendieck group of the category of finitely generated modules over $R$ and its subcategories (see 2.1, 2.2, 2.5). Our results show that the existence of NCRs still implies strong constraints on the singularities of $R$. For example, we prove that a standard graded CohenMacaulay algebra $R$ over $\mathbb{C}$ with only rational singularities outside the irrelevant ideal has a NCR only if $R$ has rational singularities (Theorem 3.11). Our proofs utilize some non-trivial results from algebraic K-theory. For surface singularities over an algebraically closed field, we observe that the existence of NCRs actually characterizes rational singularities (Corollary 3.3). This adds a new member to a long list of interesting equivalent conditions for rationality of surface singularities.

\section{NCRs and Grothendieck groups}

Throughout this note, we denote by $R$ a commutative noetherian ring, and by mod $R$ the category of finitely generated $R$-modules. We denote by $\mathrm{K}_{0}(R)$ the Grothendieck group of the abelian category $\bmod R$. When $R$ is a normal domain, we denote by $\mathrm{Cl}(R)$ the class group of $R$. Our main technical result (Theorem 2.5) given later in this section relates the existence of NCRs and certain finiteness properties of Grothendieck groups and class groups. The first application is the following, which deals with NCRs over a normal domain. 
Corollary 2.1. Let $R$ be a semilocal normal domain. If $R$ has a $N C R$, then $\mathrm{Cl}(R)$ is a finitely generated abelian group.

A typical example of a ring which has an infinitely generated class group is $R:=\mathbb{C}[[x, y, z]] /\left(x^{3}+y^{3}+z^{3}\right)$, see 3.7. In particular, $R$ has no faithful module giving rise to a NCR by the above theorem. This gives an answer to a question by Burban [5].

The second application of our main result is the following, which deals with NCRs given by modules which are locally generators on the outside of a closed set of dimension at most one.

Corollary 2.2. Let $R$ be a semilocal ring. If $M$ is an $R$-module which is locally a generator outside a closed subscheme of $\operatorname{Spec}(R)$ of dimension at most one and gives a NCR, then $\mathrm{K}_{0}(R)$ is a finitely generated abelian group.

Let us now state and prove our key technical result. Let $(-)^{*}:=\operatorname{Hom}_{R}(-, R)$. For an $R$-module $M$, let $E:=\operatorname{End}_{R}(M)$ and we denote by

$$
a_{M}: M \otimes_{E} M^{*} \rightarrow R
$$

the natural map sending $m \otimes f \in M \otimes_{E} M^{*}$ to $f(m)$.

Definition 2.3. We define the non-generating locus $\operatorname{NG}(M)$ of $M$ as the support of $\operatorname{Cok}\left(a_{M}\right)$.

For $M \in \bmod R$, we denote by $\operatorname{add}_{R} M=$ add $M$ the full subcategory of $\bmod R$ consisting of direct summands of direct sums of copies of $M$. Recall that $M \in$ $\bmod R$ is called a generator of $\bmod R$ if there exists a surjection from a direct sum of copies of $M$ to $R$, or equivalently, $R \in$ add $M$. The following observation explains the name of $\mathrm{NG}(M)$.

Proposition 2.4. $\operatorname{NG}(M)$ is the set of prime ideals $\mathfrak{p}$ such that $M_{\mathfrak{p}}$ is not a generator of $\bmod R_{\mathfrak{p}}$.

Proof. Let $\mathfrak{p}$ be a prime ideal of $R$. Then $M_{\mathfrak{p}}$ is a generator of $\bmod R_{\mathfrak{p}}$, if and only if $\sum_{f \in M_{\mathfrak{p}}^{*}} f\left(M_{\mathfrak{p}}\right)=R_{\mathfrak{p}}$, if and only if $\left(a_{M}\right)_{\mathfrak{p}}$ is surjective, if and only if $\left(\operatorname{Cok} a_{M}\right)_{\mathfrak{p}}=0$.

For a full subcategory $\mathscr{C}$ of $\bmod R$, we denote by $\langle\mathscr{C}\rangle$ the subgroup of $\mathrm{K}_{0}(R)$ generated by elements $[X]$ with $X \in \mathscr{C}$. Our main result in this section is the following:

Theorem 2.5. Let $R$ be a semilocal ring and $M$ give a NCR of $R$. Let $\mathscr{C}_{M}$ be the full subcategory of $\bmod R$ consisting of $X$ satisfying $\operatorname{supp} X \subset \mathrm{NG}(M)$. Then $\mathrm{K}_{0}(R) /\left\langle\mathscr{C}_{M}\right\rangle$ is a finitely generated abelian group.

First let us recall a well-known fact on Grothendieck groups. Let $\mathscr{C}$ be a Serre subcategory of $\bmod R$ (i.e. $\mathscr{C}$ is closed under submodules, factor modules and 
extensions), and let $(\bmod R) / \mathscr{C}$ be the quotient abelian category of $\bmod R$ [27]: The objects of $(\bmod R) / \mathscr{C}$ is the same as $\bmod R$, and the morphism set is given by

$$
\operatorname{Hom}_{(\bmod R) / \mathscr{C}}(X, Y):=\underset{X^{\prime}, Y^{\prime}}{\lim _{R}} \operatorname{Hom}_{R}\left(X^{\prime}, Y / Y^{\prime}\right)
$$

where $X^{\prime}$ and $Y^{\prime}$ run over all submodules of $X$ and $Y$ respectively such that $X / X^{\prime}$ and $Y^{\prime}$ belong to $\mathscr{C}$. In this case we have the following observation.

Proposition 2.6. [15] $\mathrm{K}_{0}((\bmod R) / \mathscr{C})$ is isomorphic to $\mathrm{K}_{0}(R) /\langle\mathscr{C}\rangle$.

We need the following general observations on generators.

Lemma 2.7. Let $M \in \bmod R$ be a generator and $E:=\operatorname{End}_{R}(M)$. Then we have the following properties.

(a) $M^{*}$ is a projective E-module.

(b) The natural map $a_{M}: M \otimes_{E} M^{*} \rightarrow R$ is an isomorphism.

Proof. (a) Since $R \in \operatorname{add}_{R} M$, we have $M^{*} \in \operatorname{add}_{E} \operatorname{Hom}_{R}(M, M)=\operatorname{add}_{E} E$.

(b) For any $X \in \bmod R$, we denote by $b_{X}: M \otimes_{E} \operatorname{Hom}_{R}(M, X) \rightarrow X$ the natural map sending $m \otimes f$ to $f(m)$. This gives a natural transformation $b: M \otimes_{E}$ $\operatorname{Hom}_{R}(M,-) \rightarrow 1_{\operatorname{modR}}$ of additive functors $\bmod R \rightarrow \bmod R$. Since $b_{M}$ is clearly an isomorphism, so is $b_{X}$ for any $X \in \operatorname{add}_{R} M$. In particular, $b_{R}$ is an isomorphism. Since $a_{M}=b_{R}$, we have the assertion.

Clearly $\mathscr{C}_{M}$ is a Serre subcategory of $\bmod R$. Let $(\bmod R) / \mathscr{C}_{M}$ be the quotient abelian category of $\bmod R$. We define a functor

$$
F: \bmod E \stackrel{\operatorname{Hom}_{E}\left(M^{*},-\right)}{\longrightarrow} \bmod R \rightarrow(\bmod R) / \mathscr{C}_{M}
$$

where $\bmod R \rightarrow(\bmod R) / \mathscr{C}_{M}$ is a natural functor.

Lemma 2.8. $F$ is an exact functor.

Proof. Let $0 \rightarrow X \rightarrow Y \rightarrow Z \rightarrow 0$ be an exact sequence in mod $E$. Applying $\operatorname{Hom}_{E}\left(M^{*},-\right)$, we have an exact sequence

$$
0 \rightarrow \operatorname{Hom}_{E}\left(M^{*}, X\right) \rightarrow \operatorname{Hom}_{E}\left(M^{*}, Y\right) \rightarrow \operatorname{Hom}_{E}\left(M^{*}, Z\right) \rightarrow \operatorname{Ext}_{E}^{1}\left(M^{*}, X\right)
$$

We only have to show $\operatorname{Ext}_{E}^{1}\left(M^{*}, X\right) \in \mathscr{C}_{M}$. For any prime ideal $\mathfrak{p} \notin \operatorname{NG}(M)$, we have that $M_{\mathfrak{p}}^{*}$ is a projective $E_{\mathfrak{p}}$-module by Lemma 2.7. Thus we have

$$
\operatorname{Ext}_{E}^{1}\left(M^{*}, X\right)_{\mathfrak{p}}=\operatorname{Ext}_{E_{\mathfrak{p}}}^{1}\left(M_{\mathfrak{p}}^{*}, X_{\mathfrak{p}}\right)=0
$$

and so $\operatorname{supp} \operatorname{Ext}_{E}^{1}\left(M^{*}, X\right) \subset \operatorname{NG}(M)$. Thus $0 \rightarrow F X \rightarrow F Y \rightarrow F Z \rightarrow 0$ is exact. 
Next we show the following property of $F$.

Lemma 2.9. $F$ is a dense functor.

Proof. For any $X \in \bmod R$, let $Y:=\operatorname{Hom}_{R}(M, X) \in \bmod E$. Then we have

$$
F(Y)=\operatorname{Hom}_{E}\left(M^{*}, \operatorname{Hom}_{R}(M, X)\right) \cong \operatorname{Hom}_{R}\left(M \otimes_{E} M^{*}, X\right) .
$$

For any prime ideal $\mathfrak{p} \notin \operatorname{NG}(M)$, we have that $M_{\mathfrak{p}}$ is a generator of $\bmod R_{\mathfrak{p}}$. Thus $\left(a_{M}\right)_{\mathfrak{p}}:\left(M \otimes_{E} M^{*}\right)_{\mathfrak{p}} \rightarrow R_{\mathfrak{p}}$ is an isomorphism by Lemma $2.7(\mathrm{~b})$. Hence the natural map

$$
\operatorname{Hom}_{R}\left(a_{M},-\right): X \rightarrow \operatorname{Hom}_{R}\left(M \otimes_{E} M^{*}, X\right)=F(Y)
$$

induced by $a_{M}$ has the kernel and the cokernel in $\mathscr{C}_{M}$. Consequently $X$ is isomorphic to $F(Y)$ in $(\bmod R) / \mathscr{C}_{M}$.

By Lemma 2.8 and Proposition 2.6, we have a homomorphism

$$
\mathrm{K}_{0}(E) \rightarrow \mathrm{K}_{0}\left((\bmod R) / \mathscr{C}_{M}\right) \cong \mathrm{K}_{0}(R) /\left\langle\mathscr{C}_{M}\right\rangle
$$

of abelian groups. This is surjective by Lemma 2.9.

Lemma 2.10. Let $R$ be a semilocal ring and $E$ a module-finite $R$-algebra. If the global dimension of $E$ is finite, then $\mathrm{K}_{0}(E)$ is finitely generated.

Proof. Since the global dimension is finite, $\mathrm{K}_{0}(E)$ is generated by indecomposable projective $E$-modules. Since $R$ is semilocal, it follows from [11, Theorem 9] that there exist only finitely many isomorphism classes of indecomposable projective $E$ modules. Thus $\mathrm{K}_{0}(E)$ is finitely generated.

The above lemma completes the proof of Theorem 2.5.

Now we prove Corollary 2.1. We need the following fact, see [7].

Proposition 2.11. Let $R$ be a normal domain and $\Phi$ be the set of prime ideals of $R$ with height at least two. Let $\mathscr{D}$ be the full subcategory of $\bmod R$ consisting of $X$ satisfying supp $X \subset \Phi$. Then $\mathrm{K}_{0}(R) /\langle\mathscr{D}\rangle$ is isomorphic to $\mathbb{Z} \oplus \mathrm{Cl}(R)$.

Assume that $M$ gives a NCR of $R$. Since $R$ is a normal domain and $M$ is a faithful $R$-module, we have that $M_{\mathfrak{p}}$ is a faithful $R_{\mathfrak{p}}$-module and hence $M_{\mathfrak{p}}$ has a non-zero free summand for any prime ideal $\mathfrak{p} \notin \Phi$. Thus we have $\mathrm{NG}(M) \subset \Phi$ and $\mathscr{C}_{M} \subset \mathscr{D}$. By Theorem 2.5, we have that $\mathrm{K}_{0}(R) /\langle\mathscr{D}\rangle$ is finitely generated. By Proposition 2.11, we have the assertion.

Finally we prove Corollary 2.2. As $R$ is semilocal and $\operatorname{dim} \operatorname{NG}(M) \leq 1$, the set $\operatorname{NG}(M)$ is finite. Since $\left\langle\mathscr{C}_{M}\right\rangle$ is generated by $R / \mathfrak{p}$ with $\mathfrak{p} \in \operatorname{NG}(M)$, it is finitely generated. Since $\mathrm{K}_{0}(R) /\left\langle\mathscr{C}_{M}\right\rangle$ is finitely generated by Theorem 2.5, so is $\mathrm{K}_{0}(R)$. 


\section{NCRs and rational singularities}

In this section let $R$ be a normal domain containing a field $k$. We wish to discuss the following:

Question 3.1. Suppose $R$ has a NCR. When can we deduce that $\operatorname{Spec}(R)$ has rational singularities?

Recall that a variety $Y$ is said to have rational singularities if for any (equivalently, some) resolution of singularity $f: X \rightarrow Y$, we have $R^{i} f_{*} \mathcal{O}_{X}=0$ for $i>0$. When $Y=\operatorname{Spec}(R)$ this reduces to $H^{i}\left(X, \mathcal{O}_{X}\right)=0$ for $i>0$ (see [31, Section 1]).

The above question is motivated by a beautiful result by Stafford and Van den Bergh ([32, Theorem 4.2]):

Theorem 3.2. (Stafford-Van den Bergh) Let $k$ be an algebraically closed field of characteristic 0 and $\Delta$ be a prime affine $k$-algebra that is finitely generated as a module over its center $Z(\Delta)$. If $\Delta$ is a non-singular $Z(\Delta)$-order then $Z(\Delta)$ has only rational singularities.

In particular, suppose $R$ is a Gorenstein normal affine $k$-algebra. If $R$ has an $N C C R$, then $\operatorname{Spec}(R)$ has only rational singularities.

In fact, at the end of their paper Stafford and Van den Bergh raised the question of whether it is enough to only assume that we have a maximal Cohen-Macaulay module giving a NCR but $R$ is not necessarily Gorenstein ([32, Question 5.2]).

Our first result shows that having rational (isolated) singularity characterizes the existence of NCRs for surface singularities. For a Cohen-Macaulay ring $R$, let $\Omega C M(R)$ denote the category of first syzygies of some maximal Cohen-Macaulay modules. Thus $\Omega C M(R)$ consists of all $X \in \bmod R$ such that there exists an exact sequence $0 \rightarrow X \rightarrow P \rightarrow Y \rightarrow 0$ with a projective $R$-module $P$ and a maximal Cohen-Macaulay $R$-module $Y$.

Corollary 3.3. Let $(R, \mathfrak{m}, k)$ be a local normal domain of dimension two. Consider the following:

(1) $\Omega C M(R)$ is of finite type (that is, there exists $M \in \bmod (R)$ such that $\Omega C M(R)=$ add $M)$.

(2) R has a NCR.

(3) $\mathrm{Cl}(R)$ is a finitely generated abelian group.

(4) $\mathrm{K}_{0}(R) \otimes_{\mathbb{Z}} \mathbb{Q}$ is a finite dimensional $\mathbb{Q}$-vector space (equivalently, $\mathrm{Cl}(R)$ has a finite rank).

(5) $\operatorname{Spec}(R)$ has rational singularities.

Then $(1) \Rightarrow(2) \Rightarrow(3) \Rightarrow(4)$. If $R$ is excellent, henselian and $k$ is algebraically closed, then (3) $\Rightarrow(5) \Rightarrow(1)$. If in addition $k$ has characteristic 0 then (4) $\Rightarrow(5)$. 
Proof. The implication (1) $\Rightarrow(2)$ is $[20$, Theorem 2.10] and (2) $\Rightarrow$ (3) is Corollary 2.1. The implication (3) $\Rightarrow$ (4) is trivial. The implication (3) $\Rightarrow$ (5) is essentially $[25,17.3]$. The proof works almost the same, except for the crucial Complement 11.3, where one needs to replace condition (2) of Theorem 1.7 in [4] by the condition that the Picard group of the curve $Z$ is finitely generated. Similarly (4) $\Rightarrow(5)$ in characteristic 0 . The statement $(5) \Rightarrow$ (1) follows from the fact that there are only finitely many indecomposable special Cohen-Macaulay modules (see [36], [20, Theorems 3.6 and 2.10]). Note that the result in [36] was stated for singularities over complex numbers, but the proof also works for our case, the extra information we need is the existence of a (minimal) desingularization of Spec $R$, which is known (cf. [25, Theorem 4.1]). One can bypass the use of Grauert-Riemenschneider vanishing used in Wunram's proof by the discussion before Theorem 5 in [13].

Example 3.4. The implication $(3) \Rightarrow(5)$ in Corollary 3.3 really requires all the assumptions. It is not true when $k$ is not algebraically closed (but $R$ is complete): Salmon ([30]) showed that $k(u)[[x, y, z]] /\left(x^{2}+y^{3}+u z^{6}\right)$ is factorial for any field $k$. The condition that $R$ is henselian is also crucial: the ring $R=k[x, y, z]_{(x, y, z)} /\left(x^{r}+\right.$ $y^{s}+z^{t}$ ) where $r, s, t$ are pairwise prime, is factorial over any field $k$ ([10, Corollary 10.17]).

Also, the implication (4) $\Rightarrow(5)$ may fail in positive characteristics. In fact, when $k=\overline{\mathbb{F}_{p}}$ the class group will always be locally finite.

Now we discuss Question 3.1 in higher dimension. We may assume $R$ is a complete local ring to study Question 3.1 by the following:

Lemma 3.5. Suppose $M$ gives a NCR of $R$ and $\mathfrak{p} \in \operatorname{Spec}(R)$. Then $M_{\mathfrak{p}}$ gives a NCR of $R_{\mathfrak{p}}$. If $(R, \mathfrak{m})$ is local then the completion $\hat{M}$ of $M$ gives a NCR of $\hat{R}$.

Proof. Let $\Lambda$ be a module-finite $R$-algebra. We denote by f.1. $\Lambda$ the category of $\Lambda$-modules of finite length. We only have to show gl. $\operatorname{dim}\left(S \otimes_{R} \Lambda\right) \leq \operatorname{gl} \operatorname{dim} \Lambda$ for $S:=R_{\mathfrak{p}}$ or $S:=\hat{R}$.

When $S=R_{\mathfrak{p}}$ (respectively, $S=\hat{R}$ ), there is an exact dense functor $S \otimes_{R}-$ : $\bmod \Lambda \rightarrow \bmod \left(S \otimes_{R} \Lambda\right)$ (respectively, $S \otimes_{R}-:$ f.1. $\Lambda \rightarrow$ f.l. $\left(S \otimes_{R} \Lambda\right)$ ). In particular we have proj. $\operatorname{dim}_{S \otimes_{R} \Lambda}\left(S \otimes_{R} M\right) \leq$ proj. $\operatorname{dim}_{\Lambda} M$. Since gl. $\operatorname{dim} \Lambda=$ $\sup _{X \in \bmod \Lambda}\left\{\right.$ proj. $\left.\operatorname{dim}_{\Lambda} X\right\}=\sup _{X \in \text { f.l. } \Lambda}\left\{\right.$ proj. $\left.\operatorname{dim}_{\Lambda} X\right\}$, the assertion follows.

The next result shows that NCRs also behave well under separable field extensions.

Lemma 3.6. Let $R$ be a commutative algebra over a field $K$ and $L$ a separable field extension of $K$. If $M$ gives a NCR of $R$, then $L \otimes_{K} M$ gives a NCR of $L \otimes_{K} R$.

Proof. Let $E:=\operatorname{End}_{R}(M)$. Clearly we have $\operatorname{End}_{L \otimes_{K} R}\left(L \otimes_{K} M\right)=L \otimes_{K} E$. We only have to show that $L \otimes_{K} E$ has finite global dimension. For any $X \in \bmod E$, 
clearly the $L \otimes_{K} E$-module $L \otimes_{K} X$ has finite projective dimension. It is enough to show that any simple $L \otimes_{K} E$-module $S$ is a direct summand of $L \otimes_{K} X$ for some $X \in \bmod E$.

We regard $S$ as an $E$-module, and we take a simple $E$-submodule $X$ of $S$. Then we have $L X=S$, and we have a surjection $L \otimes_{K} X \rightarrow S$ of $L \otimes_{K} E$-modules sending $l \otimes x \rightarrow l x$. Since $L$ is a separable extension of $K$, we have that $L \otimes_{K} X$ is a semisimple $L \otimes_{K} E$-module [8, Corollary 7.8(ii)]. Thus $S$ is a direct summand of $L \otimes_{K} X$ and we complete the proof.

For a scheme $X$ let $\mathrm{CH}_{i}(X)$ denote the Chow group of algebraic cycles of dimension $i, C H_{i}(X)_{\mathbb{Q}}=C H_{i}(X) \otimes_{\mathbb{Z}} \mathbb{Q}$, and $\mathrm{CH}_{*}(X)$ the total Chow group. We shall need the following well-known facts, see [14, Exercise II.6.3], [12] and [22]:

Theorem 3.7. Let $A$ be the homogeneous coordinate ring of a projective variety $X$ over a field $k$ and $R$ be the local ring of $A$ at the irrelevant ideal. Let $h$ denote the class in $\mathrm{CH}^{*}(X)_{\mathbb{Q}}$.

(1) There is an exact sequence

$$
0 \rightarrow \mathbb{Z} \rightarrow \mathrm{Cl}(X) \rightarrow \mathrm{Cl}(R) \rightarrow 0
$$

where the first map sends 1 to $h$.

(2) We have the following isomorphisms of $\mathbb{Q}$-vector spaces:

$$
\mathrm{CH}_{*}(X)_{\mathbb{Q}} /\left(h \cap \mathrm{CH}_{*}(X)_{\mathbb{Q}}\right) \cong \mathrm{CH}_{*}(A)_{\mathbb{Q}} \cong \mathrm{CH}_{*}(R)_{\mathbb{Q}} \cong \mathrm{K}_{0}(R)_{\mathbb{Q}}
$$

where the first two are graded isomorphisms.

The second isomorphism in Theorem 3.7(2) is [22, Lemma 4.1] and the first isomorphism is only stated for $X$ smooth in [22, Theorem 1.3]. However we notice that the isomorphism holds without assuming $X$ smooth, $c f$. the proof of Proposition 3.10.

Before moving on we recall the definition of Serre's conditions $\left(S_{n}\right)$. For a nonnegative integer $n, M$ is said to satisfy $\left(S_{n}\right)$ if:

$$
\operatorname{depth}_{R_{p}} M_{p} \geq \min \left\{n, \operatorname{dim}\left(R_{p}\right)\right\} \forall p \in \operatorname{Spec}(R) .
$$

Proposition 3.8. Let $R$ be a normal local ring. Let $M$ be a finitely generated faithful $R$-module. Then $\operatorname{NG}(M)$ is a closed subscheme of $\operatorname{Spec}(R)$ of codimension at least 2. If in addition we assume that $\operatorname{End}_{R}(M)$ is $\left(S_{3}\right)$, then $M$ is locally free outside the singular locus $\operatorname{Sing}(R)$. In particular, $\operatorname{NG}(M) \subseteq \operatorname{Sing}(R)$.

If moreover $M$ gives a NCR and $\operatorname{dim} \operatorname{Sing}(R) \leq 1$, then $\mathrm{K}_{0}(R)$ is a finitely generated abelian group. 
Proof. The first assertion follows from the proof of Corollary 2.1. The last assertion follows from Corollary 2.2. Assume now that $\operatorname{End}_{R}(M)$ is $\left(S_{3}\right)$. The assumption

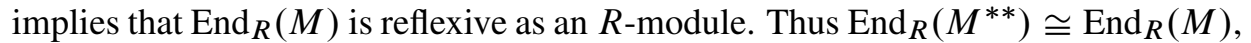
and we may assume that $M$ is reflexive. Now what we need to prove follows from the fact that if $R$ is a regular local ring and $M$ is a reflexive $R$-module, then $\operatorname{End}_{R}(M)$ is $\left(S_{3}\right)$ if and only if $M$ is free (see [16, Corollary 2.9]).

The following result is essentially due to Roitman [28]. We give a proof for the sake of completeness. Given a variety $X, \mathrm{CH}_{0}(X)_{\mathbb{Q}}$ is said to be supported in dimension $l$ if there exists an $l$-dimensional closed subvariety $Z$ of $X$ such that the proper pushforward map $\mathrm{CH}_{0}(Z)_{\mathbb{Q}} \rightarrow \mathrm{CH}_{0}(X)_{\mathbb{Q}}$ is surjective.

Lemma 3.9. Let $X$ be a smooth projective variety over $\mathbb{C}$. If $\mathrm{CH}_{0}(X)_{\mathbb{Q}}$ is supported in dimension $l$, then $H^{i}\left(X, \mathcal{O}_{X}\right)=0$ for all $i>l$.

Proof. By localization there is an $l$-dimensional subscheme $j: Z \hookrightarrow X$ such that $\mathrm{CH}_{0}(X-Z)_{\mathbb{Q}}=0$. Let $d$ be the dimension of $X$ over $\mathbb{C}$. By a result of Bloch-Srinivas on the decomposition of the diagonal [6, Proposition 1], there is a decomposition $\Delta_{X}=\Gamma_{1}+\Gamma_{2} \in \mathrm{CH}_{d}(X \times X)_{\mathbb{Q}}$. Here $\Delta_{X}$ is the class of the diagonal inside $\mathrm{CH}_{d}(X \times X)_{\mathbb{Q}}, \Gamma_{1}$ is a cycle supported on $X \times Z$ and $\Gamma_{2}$ is a cycle supported on $D \times X$ for some divisor $D$ inside $X$. Let's write $f: \widetilde{D} \rightarrow D$ for a resolution of singularities of $D$ and $g: \widetilde{Z} \rightarrow Z$ for a resolution of singularities of $Z$. Then the contravariant actions of $\Gamma_{1}$ and $\Gamma_{2}$ on $H^{i}(X, \mathbb{Q})$ are morphisms of Hodge structures. The morphism $\Gamma_{1}^{*}: H^{i}(X, \mathbb{Q}) \rightarrow H^{i}(X, \mathbb{Q})$ factors through $j^{*}: H^{i}(X, \mathbb{Q}) \rightarrow H^{i}(\widetilde{Z}, \mathbb{Q})$ and the morphism $\Gamma_{2}^{*}: H^{i}(X, \mathbb{Q}) \rightarrow H^{i}(X, \mathbb{Q})$ factors through the Gysin morphism $f_{*}: H^{i-2}(\widetilde{D}, \mathbb{Q}) \rightarrow H^{i}(X, \mathbb{Q})$. In particular, $\Gamma_{1}$ acts trivially on $H^{i}\left(X, \mathcal{O}_{X}\right)$ for $i>l$. Now, since $f_{*}$ is a morphism of Hodge structures of bidegree $(1,1)$, it follows that the intersection of the image of $f_{*}$ with $H^{i}\left(X, \mathcal{O}_{X}\right)=H^{0, i}(X)$ is zero. Thus, if $i>l$ and if $\alpha$ is any cohomology class in $H^{i}\left(X, \mathcal{O}_{X}\right)$, we have

$$
\alpha=\Delta_{X}^{*} \alpha=\Gamma_{1}^{*} \alpha+\Gamma_{2}^{*} \alpha=0
$$

i.e. $H^{i}\left(X, \mathcal{O}_{X}\right)=0$.

Proposition 3.10. Let $k$ be a field, let $R$ be a standard graded algebra over $k$, i.e. a graded Noetherian ring with $R_{0}=k$ and $R=R_{0}\left[R_{1}\right]$, of dimension at least 3. Let $\mathfrak{m}:=\bigoplus_{i>0} R_{i}, X=\operatorname{Proj} R$ and let $Z$ be a closed subscheme of codimension at least 2 in Spec $R$. Let $\mathscr{C}$ be the subcategory of $\bmod R$ generated by the finitely generated $R$-modules $M$ with supp $M \subseteq Z$. Assume $K_{0}(R) /\langle\mathscr{C}\rangle$ is finitely generated. Then $\mathrm{CH}_{0}(X)_{\mathbb{Q}}$ is supported in codimension 1.

Proof. By Riemann-Roch [12, §18], we have an isomorphism $\tau_{X}: \mathrm{K}_{0}(R)_{\mathbb{Q}} \rightarrow$ $\mathrm{CH}_{*}(R)_{\mathbb{Q}}$ which is covariant with respect to proper morphisms. The subgroup $\langle\mathscr{C}\rangle$ 
of $\mathrm{K}_{0}(R)$ is included in the image of $\mathrm{K}_{0}(Z)$ inside $\mathrm{K}_{0}(R)$ and it follows that $\mathrm{K}_{0}(R)$ is generated by $\mathrm{K}_{0}(Z)$ via the natural inclusion $Z \hookrightarrow \operatorname{Spec} R$ and by finitely many classes. Thus $\mathrm{CH}_{1}(R)_{\mathbb{Q}}$ is generated by $\mathrm{CH}_{1}(Z)_{\mathbb{Q}}$ and by finitely many 1 -cycles. Up to adding finitely many components of codimension $\geq 2$ in $\operatorname{Spec} R$ to $Z$, we may even assume that $\mathrm{CH}_{1}(R)_{\mathbb{Q}}$ is supported on $Z$.

In the proof of [22, Theorem 1.3], Kurano establishes the existence of the following exact sequence for $v>0$ (see [22, (4.16)] and notice that no smoothness assumption on $X$ is necessary for (4.16) to hold):

$$
\mathrm{CH}_{v}(X) \rightarrow \mathrm{CH}_{v-1}(X) \rightarrow \mathrm{CH}_{v}(R) \rightarrow 0 \text {. }
$$

The map on the left is given by intersecting with $h=c_{1}\left(O_{X}(1)\right)$ and the second map is the composite

$$
\mathrm{CH}_{v-1}(X) \stackrel{\eta^{*}}{\longrightarrow} \mathrm{CH}_{v}(\widetilde{X}-\{t\}) \stackrel{\left(j^{*}\right)^{-1}}{\longrightarrow} \mathrm{CH}_{v}(\widetilde{X}) \stackrel{k^{*}}{\longrightarrow} \mathrm{CH}_{v}(R) .
$$

Here $\widetilde{X}$ is the projective cone over $X,\{t\}$ is the vertex of $\widetilde{X}$ and $\operatorname{Spec} R=\widetilde{X}-X$ is the affine cone over $X$. We refer to [22] for more details. Important to us is that $k: \operatorname{Spec} R \rightarrow \widetilde{X}$ and $j: \widetilde{X}-\{t\} \rightarrow \widetilde{X}$ are open immersions and that $\eta: \widetilde{X}-\{t\} \rightarrow$ $X$ is a smooth $\mathbb{A}_{k}^{1}$-bundle. In particular these three morphisms are flat. Let $\widetilde{Z}$ be the closure of $Z$ inside $\widetilde{X}$ and let $Y$ be the image (closed by definition) in $X$ of $\left.\widetilde{Z}\right|_{\widetilde{X}-\{t\}}$ via $\eta$. By definition of flat pullbacks for Chow groups, we see that if $\mathrm{CH}_{1}(R)_{\mathbb{Q}}$ is supported on $Z$, then the composite map

$$
\mathrm{CH}_{0}(Y)_{\mathbb{Q}} \rightarrow \mathrm{CH}_{0}(X)_{\mathbb{Q}} \stackrel{k^{*}\left(j^{*}\right)^{-1}}{\longrightarrow} \eta^{*} \mathrm{CH}_{1}(R)_{\mathbb{Q}}
$$

is surjective. It follows from the short exact sequence above that $\mathrm{CH}_{0}(X)_{\mathbb{Q}}$ is supported on the union of $Y$ with a hyperplane section. It is obvious that each component of $Y$ has codimension at least one inside $X$. Therefore $\mathrm{CH}_{0}(X)_{\mathbb{Q}}$ is supported in codimension one.

Combining Proposition 3.8 and Lemma 3.9 with Theorem 2.5 and Proposition 3.10, we obtain:

Theorem 3.11. Let $R$ be a normal, Cohen-Macaulay standard graded algebra over a subfield $k$ of $\mathbb{C}$. Let $\mathfrak{m}$ be the irrelevant ideal of $R$. Suppose that $\operatorname{Spec}(R)-\{\mathfrak{m}\}$ has only rational singularities. Suppose moreover that there exists an $R$-module $M$ giving a NCR. Then $\operatorname{Spec}(R)$ has only rational singularities.

Proof. By Lemma 3.6 we can assume $k=\mathbb{C}$. Let $X=\operatorname{Proj} R$ and $d=\operatorname{dim} X$. By [35, Theorem 2.2] we only need to show that $H^{d}\left(X, \mathcal{O}_{X}(n)\right)=0$ for $n \geq 0$. It is actually enough to show that $H^{d}\left(X, \mathcal{O}_{X}\right)=0$. Indeed, letting $H$ be a hyperplane section of $X$, we have a short exact sequence for any $i$ :

$$
0 \rightarrow \mathcal{O}_{X}(i-1) \rightarrow \mathcal{O}_{X}(i) \rightarrow \mathcal{O}_{H}(i) \rightarrow 0,
$$


whose long exact sequence of cohomology gives exact sequences

$$
H^{d}\left(X, \mathcal{O}_{X}(i-1)\right) \rightarrow H^{d}\left(X, \mathcal{O}_{X}(i)\right) \rightarrow H^{d}\left(X, \mathcal{O}_{H}(i)\right)=0
$$

as $\operatorname{dim} H=d-1$. Induction on $n$ shows that $H^{d}\left(X, \mathcal{O}_{X}(n)\right)=0$ for $n \geq 0$, as desired.

Let's therefore show that $H^{d}\left(X, \mathcal{O}_{X}\right)=0$. By Proposition 3.8, NG(M) is contained in a closed subscheme of Spec $R$ of codimension 2 by normality of $R$. By Theorem 2.5 and Proposition 3.10, it follows that $\mathrm{CH}_{0}(X)_{\mathbb{Q}}$ is supported on a divisor $D$, i.e. $\mathrm{CH}_{0}(X-D)_{\mathbb{Q}}=0$. Let $f: \widetilde{X} \rightarrow X$ be a resolution of singularities of $X$ and let $\widetilde{D}=f^{-1}(D)$. Up to adding some components to $D$, we may assume that $f$ induces an isomorphism $\widetilde{X}-\widetilde{D} \rightarrow X-D$. Also we still have $\mathrm{CH}_{0}(X-D)_{\mathbb{Q}}=0$ and it follows that $\mathrm{CH}_{0}(\widetilde{X}-\widetilde{D})_{\mathbb{Q}}=0$, i.e. that $\mathrm{CH}_{0}(\widetilde{X})_{\mathbb{Q}}$ is supported on a divisor. By Lemma 3.9, we then have $H^{d}\left(\widetilde{X}, \mathcal{O}_{\widetilde{X}}\right)=0$. Since $X$ has only rational singularities, we see from the Leray-Serre spectral sequence that $H^{d}\left(X, \mathcal{O}_{X}\right)=0$.

A consequence of the first half of the proof of Theorem 3.11 and of Lemma 3.9 (applied in the case $l=0$ ) is the following

Corollary 3.12. Let $X$ be a smooth projective variety over $\mathbb{C}$. If $\operatorname{dim}_{\mathbb{Q}} \mathrm{CH}_{0}(X)_{\mathbb{Q}}<\infty$, then $X$ admits an embedding into a projective space whose homogeneous coordinate ring has only rational singularities.

In view of Proposition 3.8 and Theorem 3.7 we ask:

Question 3.13. Let $(R, \mathfrak{m}, k)$ be a Cohen-Macaulay complete local normal domain with $k$ an algebraically closed field. If $\mathrm{K}_{0}(R)$ is finitely generated, must $\operatorname{Spec}(R)$ have only rational singularities?

Corollary 3.3 shows that the answer is yes in dimension 2. Of course, in higher dimensions the existence of desingularizations is not known for positive or mixed characteristics, so one may need to replace the condition of rational singularities with suitable concepts such as being $F$-rational or pseudo-rational.

Our last example illustrates some subtlety involving Lemma 3.9.

Example 3.14. Lemma 3.9 might not be true over fields of characteristic 0 whose transcendance degree over their prime subfield is not large enough. Indeed, consider a K3-surface $X$ over the algebraic closure of $\mathbb{Q}$. Then, $H^{2}\left(X, \mathcal{O}_{X}\right)$ is not zero but it is expected (as part of the Bloch-Beilinson conjectures) that $\mathrm{CH}_{0}(X)_{\mathbb{Q}}=\mathbb{Q}$.

Provided such an example exists, it could potentially yield a negative example to Question 3.13. Note that $\mathrm{CH}(X)_{\mathbb{Q}}$ is a finite dimensional $\mathbb{Q}$-vector space (it is known that the rank of the Picard group of $X$ is finite). We can use an very ample line bundle on $X$ to embed $X$ into some projective space. Let $R$ be the homogeneous coordinate ring of such an embedding, thus $\operatorname{dim}_{\mathbb{Q}} \mathrm{CH}(R)_{\mathbb{Q}}<\infty$. But if $R$ has rational singularities, then $H^{2}\left(X, \mathcal{O}_{X}\right)=0$ (cf. [35, Theorem 2.2] and [34]). 
Acknowledgements. The first author thanks Joseph Lipman and Karl Schwede for some helpful conversations on rational singularities. The second author thanks Michael Wemyss for valuable discussions. This work started when the second and third authors visited University of Kansas in August, 2011. They are grateful for the hospitality.

\section{References}

[1] M. Auslander, Functors and morphisms determined by objects, Representation theory of algebras (Proc. Conf., Temple Univ., Philadelphia, Pa., 1976), pp. 1-244. Lecture Notes in Pure Appl. Math., Vol. 37, Dekker, New York, 1978. Zbl 0383.16015 MR 480688

[2] M. Auslander, Isolated singularities and existence of almost split sequences, In Representation Theory II. Lecture Notes in Mathematics 1178, Springer-Verlag, BerlinHeidelberg, 1986, 194-242. Zbl 0633.13007 MR 842486

[3] M. Auslander, O. Goldman, Maximal Orders, Trans. Amer. Math. Soc. 97 (1960), 1-24. Zbl 0117.02506 MR 117252

[4] M. Artin, Some Numerical Criteria for Contractability of Curves on Algebraic Surfaces, Amer. J. Math. (3) 84 (1962), 485-496. Zbl 0105.14404 MR 146182

[5] I. Burban, Private communication.

[6] S. Bloch, V. Srinivas, Remarks on correspondences and algebraic cycles, Amer. J. Math. (5) 105 (1983), 1235-1253. Zbl 0525.14003 MR 714776

[7] C.-Y. J. Chan, Filtrations of modules, the Chow group, and the Grothendieck group, J. Algebra 219 (1999), 330-344. Zbl 0949.13008 MR 1707674

[8] C. W. Curtis, I. Reiner, Methods of representation theory. Vol. I. With applications to finite groups and orders, reprint of the 1981 original, Wiley Classics Library. A WileyInterscience Publication. John Wiley \& Sons, Inc., New York, 1990. Zbl 0698.20001 MR 1038525

[9] H. Dao, Some observations on local and projective hypersurfaces, Math. Res. Lett. 15 (2008), 207-219. Zbl 1229.13014 MR 2385635

[10] R. M. Fossum, The divisor class group of a Krull domain, Ergebnisse der Mathematik und ihrer Grenzgebiete, Band 74, Springer-Verlag, New York-Heidelberg, 1973. Zbl 0256.13001 MR 382254

[11] K. R. Fuller, W. A. Shutters, Projective modules over non-commutative semilocal rings, Tohoku Math. J. (2) 27 (1975), no. 3, 303-311. Zbl 0316.16026 MR 435142

[12] W. Fulton, Intersection Theory, Springer-Verlag, Berlin (1998). Zbl 0885.14002 MR 1644323

[13] V. Gavran, Kahn's correspondence and Cohen-Macaulay modules over abstract surface and curve singularities, Journal of Singularities 4 (2012), 68-73. Zbl 1292.13003 MR 3044487

[14] R. Hartshorne, Algebraic Geometry, Graduate Text in Mathematics, Springer-Verlag, New York, (1977). Zbl 0367.14001 MR 463157 
[15] A. Heller, Some exact sequences in algebraic K-theory, Topology 4 (1965), 389-408. Zbl 0161.01507 MR 179229

[16] C. Huneke, R. Wiegand, Tensor products of modules, rigidity and local cohomology, Math. Scand. 81 (1997), 161-183. Zbl 0908.13010 MR 1612887

[17] O. Iyama, Rejective subcategories of artin algebras and orders, arXiv:math/0311281, 2003.

[18] O. Iyama, Higher-dimensional Auslander-Reiten theory on maximal orthogonal subcategories, Adv. Math. 210 (2007), no. 1, 22-50. Zbl 1115.16005 MR 2298819

[19] O. Iyama, Auslander correspondence, Adv. Math. 210 (2007), no. 1, 51-82. Zbl 1115.16006 MR 2298820

[20] O. Iyama, M. Wemyss, The classification of special Cohen-Macaulay modules, Math. Z. 265 (2010), no. 1, 41-83. Zbl 1192.13012 MR 2606949

[21] O. Iyama, M. Wemyss, Maximal modifications and Auslander-Reiten duality for non-isolated singularities, Invent. Math. 197 (2014), no. 3, 521-586. Zbl 06358026 MR 3251829

[22] K. Kurano, A remark on the Riemann-Roch formula for affine scheme associated with Noetherian local ring, Tohoku Math J. 48 (1996), 121-138. Zbl 0882.14002 MR 1373176

[23] K. Kurano, Numerical equivalence defined on Chow groups of Notherian local rings, Invent. Math. 157 (2004), 575-619. Zbl 1070.14007 MR 2092770

[24] G. J. Leuschke, Non-commutative crepant resolutions: scenes from categorical geometry, Progress in commutative algebra 1, 293-361, de Gruyter, Berlin, 2012. Zbl 1254.13001 MR 2932589

[25] J. Lipman, Rational singularities with applications to algebraic surfaces and unique factorization, Publ. Math. I.H.E.S. 36 (1969), 195-279. Zbl 0181.48903 MR 276239

[26] D. Mumford, Rational equivalence of 0-cycles on surfaces, J. Math. Kyoto. Univ. 9 (1968), 195-204. MR 249428

[27] N. Popescu, Abelian categories with applications to rings and modules, London Mathematical Society Monographs, No. 3. Academic Press, London-New York, 1973. Zbl 0271.18006 MR 340375

[28] A. A. Roitman, Rational equivalence of 0-cycles, Math. USSR Sbornik, 18 (1972), 571588.

[29] I. Reiten, M. Van den Bergh, Two-dimensional tame and maximal orders of finite representation type, Memoirs Amer. Math. Soc. 80 (1989), no. 408. Zbl 0677.16002 MR 978602

[30] P. Salmon, Su un problema posto da P. Samuel, Atti Accad. Naz. Lincei Rend. Cl. Sci. Fis. Mat. Natur. (8) 40 (1966), 801-803. Zbl 0147.01601 MR 207744

[31] K. E. Smith, F-Rational Rings have Rational Singularities, Amer. J. Math. 119 (1997), 159-180. Zbl 0910.13004 MR 1428062

[32] J. T. Stafford, M. Van den Bergh, Noncommutative resolutions and rational singularities, Mich. Math. J. 57 (2008), 659-674. Zbl 1177.14026 MR 2492474 
[33] M. Van den Bergh, Non-commutative crepant resolutions, The legacy of Niels Henrik Abel, Springer, Berlin, 2004, 749-770. Zbl 1082.14005 MR 2077594

[34] K.-i. Watanabe, Some remarks concerning Demazure's construction of normal graded rings, Nagoya Math. J. 83 (1981), 203-211. Zbl 0518.13003 MR 632654

[35] K.-i. Watanabe, Rational singularities with $k^{*}$-action, Commutative Algebra (Trento 1981), Lecture Notes in Pure and Appl. Math. 84, Marcel Dekker, New York (1983), 339-351. Zbl 0525.14001 MR 686954

[36] J. Wunram, Reflexive modules on quotient surface singularities, Math. Ann. 279 (1988) no. 4, 583-598. Zbl 0616.14001 MR 926422

[37] Y. Yoshino, Cohen-Macaulay modules over Cohen-Macaulay rings, London Mathematical Society Lecture Note Series, 146, Cambridge University Press, Cambridge, 1990. Zbl 0745.13003 MR 1079937

Received 20 July, 2012; revised 06 August, 2013

H. Dao, Department of Mathematics, University of Kansas, Lawrence, KS 66045-7523, USA

E-mail: hdao@math.ku.edu

O. Iyama, Graduate School of Mathematics, Nagoya University, Furocho, Chikusaku, Nagoya 464-8602, Japan

E-mail: iyama@math.nagoya-u.ac.jp

R. Takahashi, Graduate School of Mathematics, Nagoya University, Furocho, Chikusaku, Nagoya 464-8602, Japan;

Department of Mathematics, University of Nebraska, Lincoln, NE 68588-0130, USA

E-mail: takahashi@math.nagoya-u.ac.jp

C. Vial, DPMMS, University of Cambridge, Wilberforce Road, Cambridge, CB3 0WB, UK

E-mail: c.vial@dpmms.cam.ac.uk 